
\title{
Regionalpolitik neu denken
}

\begin{abstract}
Auf der Konferenz „Regionalpolitik neu denken“, die der Wirtschaftsdienst gemeinsam mit dem Institut der deutschen Wirtschaft am 30. Oktober 2018 in Berlin veranstaltet hat, wurden innovative Ansätze diskutiert, wie Regionalpolitik neu gestaltet werden kann. Diese wurde lange Zeit wenig beachtet, betont Michael Hüther in der Keynote der Konferenz. Allerdings hat sich dies nicht zuletzt mit der im Herbst 2018 durch die Bundesregierung einberufenen Kommission "Gleichwertige Lebensverhältnisse“ gewandelt. Das zunehmende Interesse lässt sich darauf zurückführen, dass regionale wirtschaftliche Unterschiede weitreichende Folgen für die ökonomische Prosperität und damit auch für den gesellschaftlichen Zusammenhalt haben. Hüther verweist auf die Vielschichtigkeit der Regionalpolitik und fordert eine Regionalisierung der Regionalpolitik, wobei die Ertüchtigung der Kommunalfinanzen für ihn zentral ist.
\end{abstract}

In der ersten Session „Regionen - Entwicklungsdynamik und Handlungsbedarf" zeigt Michael Pflüger auf, dass Städte und ländliche Regionen sich ungleich entwickeln und ein weiteres Aufreißen der Lücken zwischen Stadt und Land und zwischen Gewinner- und Verliererregionen zu befürchten ist. Joachim Ragnitz weist auf Unterschiede innerhalb Deutschlands hin. Er unterscheidet dabei die regionalen Disparitäten im Bereich der öffentlichen Daseinsvorsorge von denen aus wirtschaftlicher Sicht. Es zeigt sich, dass die öffentlichen Daseinsvorsorgeleistungen relativ ausgewogen sind, die unterschiedliche regionale Wirtschaftskraft jedoch ein Problem in Deutschland darstellt. Thomas Terfrüchte benennt die Gewährleistung gleichwertiger Lebensverhältnisse in den Teilräumen Deutschlands und eine nachhaltige Raumentwicklung als die zentrale Leitvorstellung der Raumordnung. Zu diskutieren ist, welche Rolle der Raumordnung mit ihrem Instrumentarium zukommt und welchen Beitrag die Regionalpolitik leisten kann.

Dr. Brigitte Preissl ist Chefredakteurin von Wirtschaftsdienst und Intereconomics in der ZBW - Leibniz-Informationszentrum Wirtschaft in Hamburg. Kristin Biesenbender, M.A., und Cora Wacker-Theodorakopoulos, Dipl.-Volkswirtin, sind dort Redakteurinnen.
Annekatrin Niebuhr betrachtet zu Beginn der zweiten Session „Herausforderungen der Regionalpolitik" regionale Disparitäten in Deutschland, die von einer Abwanderung aus strukturschwachen Regionen begleitet werden. Sie empfiehlt eine Koordinierung der regionalpolitischen Förderung mit arbeitsmarktpolitischen Maßnahmen. Martin Junkernheinrich stellt fest, dass nachdem in den letzten Jahrzehnten der „Aufbau Ost“ ein zentraler Schwerpunkt war, sich nunmehr eine gesamtdeutsche Diskussion, die auch die Abkoppelung finanzschwacher Städte in Westdeutschland einschließt, öffnet. Damit gewinnt die fiskalische Seite der Gleichwertigkeit an Bedeutung. Martin Gornig beklagt, dass die Infrastrukturinvestitionen seit Jahren sinken. Insbesondere die Gemeinden hatten erhebliche Finanzprobleme in der Vergangenheit. Dadurch sind auch Planungs- und Genehmigungskapazitäten verloren gegangen. Es sollten wieder stabile finanzielle Rahmenbedingungen geschaffen werden.

Klaus-Heiner Röhl eröffnet die dritte Session „Regionen im Wandel" mit den regionalen Fördermaßnahmen von Land, Bund und EU für das Ruhrgebiet und kommt zu dem ernüchternden Ergebnis, dass die Regionalpolitik keine Trendwende bewirkt hat. Die Region nahm nicht am Aufschwung der urbanen Räume seit der Jahrtausendwende teil; sie verlor Einwohner und fällt in der Wirtschaftskraft hinter den anderen großen Agglomerationen des Landes zurück. Peter Dehne bewertet die Daseinsvorsorge als eine wesentliche Grundlage für gleichwertige Lebensverhältnisse an jedem Ort. Er betrachtet die Daseinsvorsorge als gesellschaftliche Aufgabe und betont die Vielfalt und die besondere Verantwortung der Kommunen. Annaflavia Bianchi konzentriert sich auf die jüngsten Entwicklungen in der Emilia-Romagna, insbesondere auf den „patto per il lavoro" (Pakt für Arbeit) von 2015. Sie plädiert für einen integrierten Ansatz der Industriepolitik auf regionaler Ebene.

Raphael L'Hoest betont in der abschließenden Podiumsdiskussion „Beitrag der Regionalpolitik zur Stabilisierung der Wirtschaft in Deutschland", dass neben dem Ost-West-Gefälle auch ein Nord-Süd-Gefälle existiert. Um gleichwertige Lebensverhältnisse zu gewährleisten, kommt der Bund-Länder-Gemeinschaftsaufgabe „Verbesserung der regionalen Wirtschaftsstruktur" im Rahmen einer regelgebundenen Regionalpolitik eine zentrale Bedeutung zu. Martin Rosenfeld hält es für sinnvoll, ärmere Regionen und Kommunen in die Lage zu versetzen, eigene Strategien für den Aufholprozess zu entwickeln und umzusetzen und nicht zentral zu verordnen. 